\title{
Quality of Service Comparison of LOS and NLOS Propagation in a LoRaWAN- Based Remote Monitoring System
}

\author{
Fikri Nizar Gustiyana, Muntaqo Alfin Amanaf, Danny Kurnianto* \\ Institut Teknologi Telkom Purwokerto, 128 D.I Panjaitan Street, Purwokerto, Indonesia
}

\section{ARTICLE INFORMATION}

Received: May 10, 2020

Revised: July 14, 2021

Available online: July 28, 2021

\section{KEYWORDS}

LoRa, LoRaWAN, Wireless, Internet Of

Things

\section{CORRESPONDENCE}

Phone: -

E-mail: dannykurnianto@ittelkom-pwt.ac.id

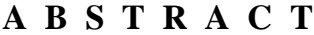

\begin{abstract}
Indonesia is an agricultural country with a wealth of natural resources. The agricultural secto is one of the natural resources with great potential. The processing and monitoring of very large agricultural land is currently a problem that must be resolved as soon as possible. The use of a remote monitoring system is the solution to this problem. The LoRa protocol is one of the communication protocols that can be used on large farms. This communication system is part of the low-power wide-area network communication system. The LoRaWAN communication system was implemented on agricultural soil moisture monitoring devices in this study. Based on the findings, it is possible to conclude that the propagation used at the time of transmission influences the success rate of data transmission via LoRaWAN communication. Line of Sight (LOS) propagation has a higher success rate than Non Line of Sight (NLOS) propagation. The LOS value is $17 \%$ greater than the NLOS at a distance of 100 meters. The LOS value is $24 \%$ greater than the NLOS at a distance of 150 meters. The LOS value is $3 \%$ greater than the NLOS value at a distance of 200 meters. LOS propagation measurement throughput is higher than NLOS propagation measurement throughput
\end{abstract}

\section{INTRODUCTION}

Agriculture is one of the many fields where information and communication technology has been used. Indonesia is an agricultural country with a wealth of natural resources. With a population of around 38.70 million people, the percentage in the agricultural, forestry, and fishery sectors is 30.46 percent [1]. Soil is one of the most important factors in agriculture that must be taken into account as thoroughly as possible in order to produce the best results. One method is to use a remote monitoring system [2][11].

Long-distance monitoring of agricultural land can be accomplished using communication technology. LoRaWAN technology is a low-power communication technology that can be used for agricultural land monitoring. LoRaWAN technology includes important features like data encryption and end-to-end device security.LoRaWAN gateways can cover a large number of end devices over a long distance [3][4].

Several other researchers have conducted studies on Lora and LoRaWAN communication, including fishing boat monitoring systems [5,] comparisons of LoRa, Sigfox, and NB-IoT communications [6,] LoRa in Star Topology [7], LoRa for Wireless Sensor Network [8,] and LoRa for Energy Harvesting [9]. In a wireless communication system, information sent from the sender to the receiver must be properly maintained so that no https://doi.org/10.25077/jnte.v10n2.781.2021 significant errors occur at the receiver. Obstacles (NLOS) are one of the causes of information transmission errors in wireless communication systems [10]. The purpose of this study is to compare the quality of communication services provided by LOS and NLOS propagation. Success rate and throughput are the service quality parameters to be compared.

\section{METHOD}

This research makes use of a network on a soil moisture monitoring device that is based on LoRaWAN communication tools. The network's performance is assessed by making a comparison of data from networks deployed in LOS propagation zones with networks located in NLOS areas. The Quality of Service (QoS) parameters, namely success rate and throughput, are referred to as network performance.Figure 1 shows a simple LoRaWAN communication architecture comprised of end devices, gateways, network servers, and application servers.

According to Figure 1, the end device uses the LoRa communication protocol to send data in the form of soil moisture values read from the sensor to the gateway. The gateway communicates with the network server via a wireless communication interface, specifically Wi-Fi. LoRa.id, the target network server, has been directly integrated with the Antares.id 
application server. Furthermore, sensor data stored on the application server is displayed on an Android app created with App Inventor.

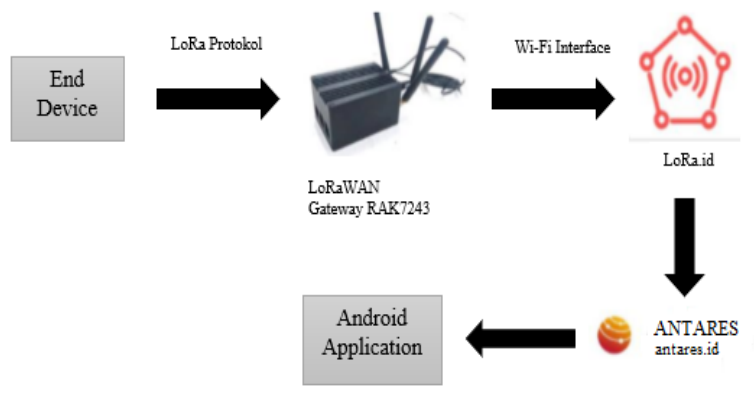

Figure 1. LoRaWAN Communication Architecture

According to Figure 1, the end device uses the LoRa communication protocol to send data in the form of soil moisture values read from the sensor to the gateway. The gateway communicates with the network server via a wireless communication interface, specifically Wi-Fi. LoRa.id, the target network server, has been directly integrated with the Antares.id application server. Furthermore, sensor data stored on the application server is displayed on an Android app created with App Inventor. The test is divided into four scenarios: testing the success rate on LOS and NLOS propagation, testing throughput on LOS and NLOS propagation.

LOS propagation tests were conducted in the Telkom Terpadu Education Area, Purwokerto, with test distances of 100 m, 150 m, and $200 \mathrm{~m}$ without obstacles between the packet delivery paths from end devices to gateways. Figure 2 depicts the location of the end device at the time of measurement.

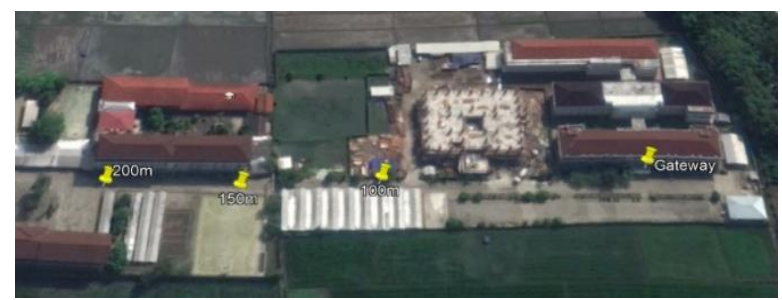

Figure 2. LOS Propagation Test Sites

NLOS propagation tests were conducted in the Telkom Integrated Education Area, Purwokerto, and the Pancurawis Rice Fields, both in Purwokerto, Central Java. The test distance varies between $100 \mathrm{~m}, 150 \mathrm{~m}$, and $200 \mathrm{~m}$, with DC, IOT, and TT class buildings acting as obstacles in the packet delivery path from the end device to the gateway. Figure 3 depicts the location of the end device at the time of measurement.

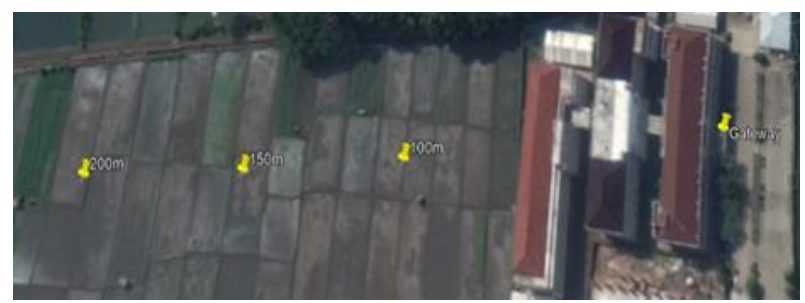

Figure 3. NLOS Propagation Test Sites
The success rate test is performed by sending 50 data packets containing soil moisture values to the antares.id platform in one measurement. The number of measurements taken ten times in a single distance. The transmission distances between the end device and the Gateway are $100 \mathrm{~m}, 150 \mathrm{~m}$, and $200 \mathrm{~m}$, respectively. The success rate is calculated by dividing the number of received packets by the number of sent packets. The better the communication transmission, the higher the success rate. The formula for calculating the success rate is shown in (1).

Succes rate $=\frac{\text { Number of received packets }}{\text { Number of sent packets }} \times 100(\%)$

Throughput testing is performed by sending packets of 10 bytes, 25 bytes, and 40 bytes for one minute without any configuration delay, and then dividing the number of packets received by the amount of time used for measurement to produce units of bits per second. The distance used in the throughput measurement is the same as the distance used in the success rate measurement, which is $100 \mathrm{~m}, 150 \mathrm{~m}$, and $200 \mathrm{~m}$. The equation used to calculate the throughput value is shown in (2).

Throughput $=\frac{\text { received packet size }(\text { bits })}{\text { delivery duration }(\text { second })}($ bit/detik)

The hop frequency method with 8 different frequency channels is used to send packets from end devices. In other words, depending on the number of frequency channels used, each transmission uses a different frequency. The frequency configured on the end device is shown in Table 1.

Table 1. Configuration of frequency channels on End Device

\begin{tabular}{cc}
\hline Channel & Frequency \\
\hline 0 & $921.1 \mathrm{Mhz}$ \\
1 & $921.3 \mathrm{Mhz}$ \\
2 & $921.5 \mathrm{Mhz}$ \\
3 & $921.7 \mathrm{Mhz}$ \\
4 & $921.9 \mathrm{Mhz}$ \\
5 & $922.1 \mathrm{Mhz}$ \\
6 & $922.3 \mathrm{Mhz}$ \\
7 & $922.5 \mathrm{Mhz}$ \\
\hline
\end{tabular}

The frequency used to receive data at the gateway must be the same as the frequency used to send data from the end device. The Gateway RAK7243 has a receiver channel specification of up to 8 channels. Table 2 shows the gateway frequency channel configuration using two center frequencies.

Table 2. Frequency channel configuration on the gateway

\begin{tabular}{ccc}
\hline Channel & Radio & Frequency \\
\hline 0 & Radio 0 (921.5 Mhz) & $921.1 \mathrm{Mhz}$ \\
1 & Radio 0 (921.5 Mhz) & $921.3 \mathrm{Mhz}$ \\
2 & Radio 0 (921.5 Mhz) & $921.5 \mathrm{Mhz}$ \\
3 & Radio 0 (921.5 Mhz) & $921.7 \mathrm{Mhz}$ \\
4 & Radio 0 (921.5 Mhz) & $921.9 \mathrm{Mhz}$ \\
5 & Radio 1 (922.5 Mhz) & $922.1 \mathrm{Mhz}$ \\
6 & Radio 1 (922.5 Mhz) & $922.3 \mathrm{Mhz}$ \\
7 & Radio 1 (922.5 Mhz) & $922.5 \mathrm{Mhz}$ \\
\hline
\end{tabular}


According to Table 2, each channel differs by $200 \mathrm{kHz}$ from the next or previous channel. Then, as shown in Table 3, for the illustration of package delivery.

Table 3. Illustration of shipping with counter

\begin{tabular}{ccc}
\hline Counter to n & Channel & Frequency \\
\hline 0 & 0 & $921.1 \mathrm{Mhz}$ \\
1 & 1 & $921.3 \mathrm{Mhz}$ \\
2 & 2 & $921.5 \mathrm{Mhz}$ \\
3 & 3 & $921.7 \mathrm{Mhz}$ \\
4 & 4 & $921.9 \mathrm{Mhz}$ \\
5 & 5 & $922.1 \mathrm{Mhz}$ \\
6 & 6 & $922.3 \mathrm{Mhz}$ \\
7 & 7 & $922.5 \mathrm{Mhz}$ \\
8 & 0 & $921.1 \mathrm{Mhz}$ \\
9 & 1 & $921.3 \mathrm{Mhz}$ \\
\hline
\end{tabular}

\section{RESULTS AND DISCUSSION}

\section{Testing Success Rate on LOS Propagation}

The following are the results of a success rate test performed by sending 50 packets 10 times with a size of 13 Byte at a distance of $100 \mathrm{~m}$; the results of the success rate test are depicted in Figure 4.

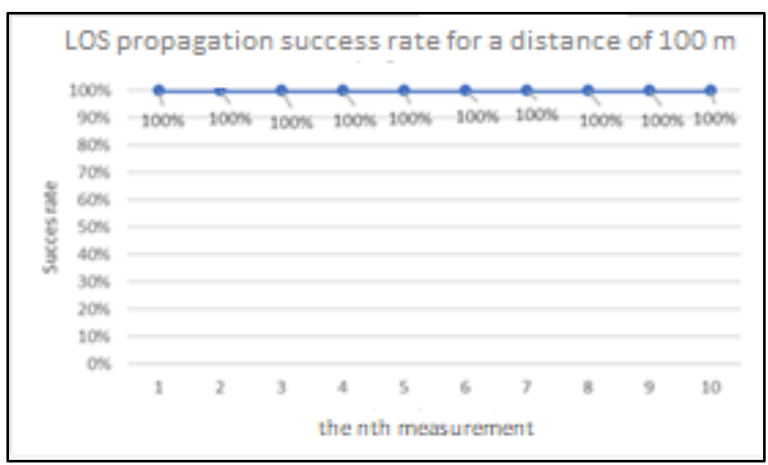

Figure 4. Graph of Success Rate on LOS Propagation Measurements at a $100 \mathrm{~m}$

According to Figure 4, the success rate measurement for LOS propagation at a distance of $100 \mathrm{~m}$ has a constant percentage value of 100 percent, indicating that all packets sent from end devices can be received by the Gateway. The average success rate from ten trials was 100 percent. Figure 5 shows the results of the success rate test by sending 50 packages 10 times at a distance of $150 \mathrm{~m}$.

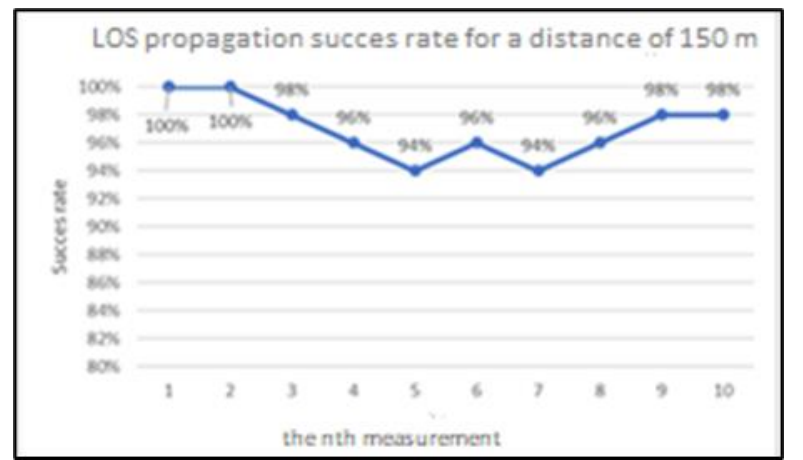

Figure 5. Graph of Success Rate on LOS Propagation Measurement at a $150 \mathrm{~m}$
According to Figure 5, the results of the success rate measurement on LOS propagation at a distance of $150 \mathrm{~m}$ have a variable percentage value. The success rate value decreased by $2 \%$ on the third, fourth, fifth, and seventh measurements compared to the previous measurement results. However, the success rate increased by $2 \%$ from the previous experiment's results at the sixth, eighth, and ninth measurements. The average success rate from ten trials was 97 percent.

Figure 6 depicts the results of measuring the success rate with the delivery of 50 packages performed ten times at a distance of 200 $\mathrm{m}$.

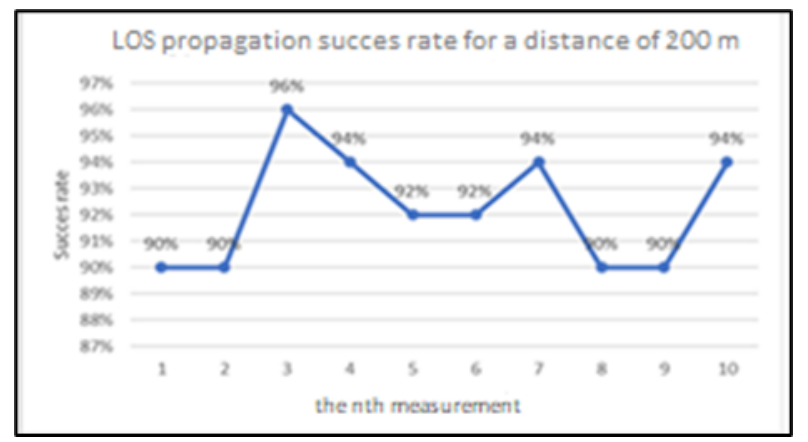

Figure 6. Success Rate Graph on LOS Propagation Measurement at a $200 \mathrm{~m}$

Figure 6 shows that the success rate in LOS propagation measurements at a distance of $200 \mathrm{~m}$ varies when compared to a distance of $150 \mathrm{~m}$. The success rate decreased by $2 \%$ in the fourth and fifth measurements and by $4 \%$ in the eighth measurement compared to the previous experiment. However, the success rate increased from the previous measurement results in the third, seventh, and tenth experiments. The average success rate from ten measurements was 92 percent. The average success rate at each distance can be calculated using the measurement results at each distance, as shown in Figure 7.

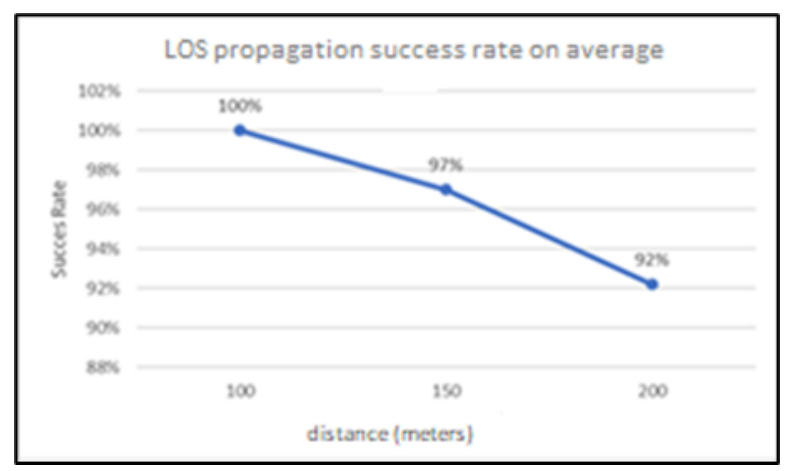

Figure 7. Graph of Average Success Rate on LOS Propagation Measurement

Based on Figure 7, it is possible to conclude that the distance of data transmission from the End Device to the Gateway affects the success rate of packet delivery in the LoRaWAN protocol of LOS propagation. The greater the distance between the End device and the Gateway, the lower the Success rate value in packet delivery, though this difference is minor. It could be caused by transmission attenuation. 


\section{Testing Success Rate on NLOS Propagation}

The success rate was determined by sending 50 packets 10 times with a size of 13 bytes at distances of $100 \mathrm{~m}, 150 \mathrm{~m}$, and $200 \mathrm{~m}$ at locations with NLOS propagation. Figure 8 depicts the success rate value at a distance of $100 \mathrm{~m}$

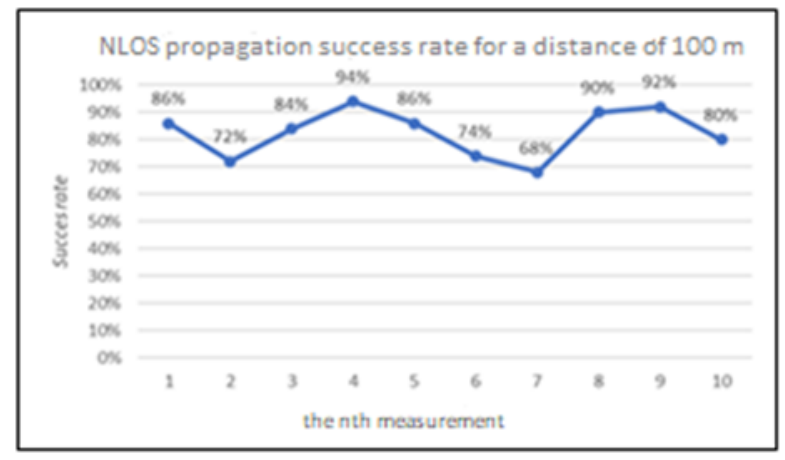

Figure 8. Success Rate Graph on NLOS Propagation Measurement at a $100 \mathrm{~m}$

According to Figure 8, the success rate in NLOS propagation measurements over a distance of $100 \mathrm{~m}$ varies. The average success rate from ten measurements was 83 percent. Figure 9 shows the value of the success rate with sending 50 packages of 10 measurements at a distance of $150 \mathrm{~m}$.

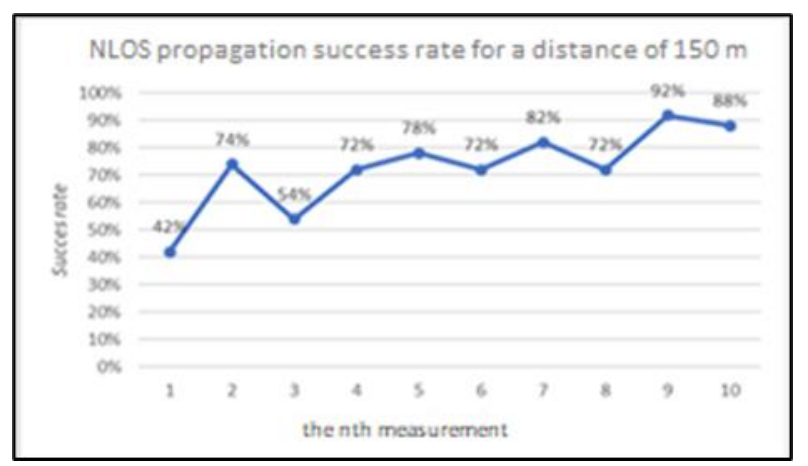

Figure 9. Graph of Success Rate on NLOS Propagation Measurements at a $150 \mathrm{~m}$

According to Figure 9, the success rate in NLOS propagation measurements over a distance of $150 \mathrm{~m}$ varies. The value decreased from the previous experiment in the third, sixth, eighth, and tenth experiments. There was a $20 \%$ decrease from the second experiment in the third experiment. It decreased by $6 \%$ from the previous experiment in the sixth experiment. Then, in the eighth experiment, the decrease was $10 \%$ less than in the seventh experiment.

It decreased by $4 \%$ in the tenth experiment compared to the ninth experiment. However, the percentage value increased from the previous experiment in the second, fourth, fifth, seventh, and ninth experiments. The second experiment increased by $32 \%$ when compared to the first. There was an $18 \%$ increase from the third experiment in the fourth experiment. Then, in the fifth experiment, there was a $6 \%$ increase from the fourth experiment. Then, in the seventh experiment, there was a $10 \%$ increase from the sixth experiment. In the ninth experiment, there was a $20 \%$ increase over the eighth experiment. The average success rate from ten trials was $73 \%$. Figure 10 depicts the success rate value obtained from the measurement at a distance of $200 \mathrm{~m}$.

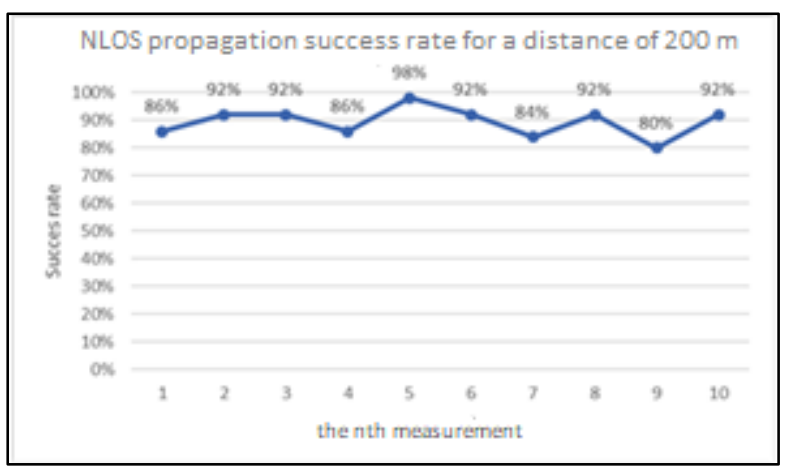

Figure 10. Graph of Success Rate on NLOS Propagation Measurements at a $200 \mathrm{~m}$

Figure 10 shows that the success rate in NLOS propagation measurements with a distance of 200 meters has varying values. The value decreased from the previous experiment in the fourth, sixth, seventh, and ninth experiments. There was a $6 \%$ decrease from the third experiment in the fourth experiment. It decreased by $6 \%$ from the previous experiment in the sixth experiment. Then, in the seventh experiment, it decreased by $8 \%$ from the sixth. And the ninth experiment decreased by $13 \%$ compared to the eighth experiment.

However, the percentage value increased from the previous experiment's results in the second, fifth, eighth, and tenth experiments. The second experiment increased by $6 \%$ when compared to the first. There was a $12 \%$ increase from the fourth experiment in the fifth experiment. Then, in the eighth experiment, there was an increase of $8 \%$ over the seventh experiment.

Then, in the tenth experiment, there was a $12 \%$ increase over the ninth experiment. The average success rate from ten trials was 89 percent. The average success rate at each distance can be calculated using the measurement results at each distance, as shown in Figure 11.

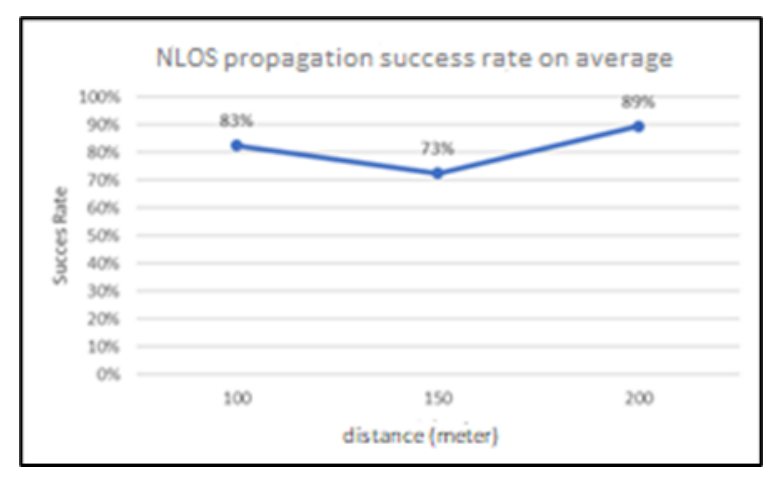

Figure 11. Graph of Average Success Rate on NLOS Propagation Measurements

Figure 11 shows that the success rate of NLOS propagation does not change significantly when backfilling is done at $100 \mathrm{~m}, 150$ $\mathrm{m}$, and $200 \mathrm{~m}$ distances.

\section{Testing Throughput on LOS Propagation}

Throughput on LOS propagation is measured at 100, 150, and 200 meter distances by sending as much data as possible from the end 
device to the Application Server in 1 minute with packet sizes of 10, 25, and 40 Bytes. The measurement results at each distance are then converted into bits and divided by the measurement time, which is 1 minute or 60 seconds, to obtain the final result in bits per second. Figure 12 depicts the throughput test results.

According to the graph in Figure 12, the larger the packet sent, the higher the Throughput generated. The lower the throughput produced, the greater the measurement distance.

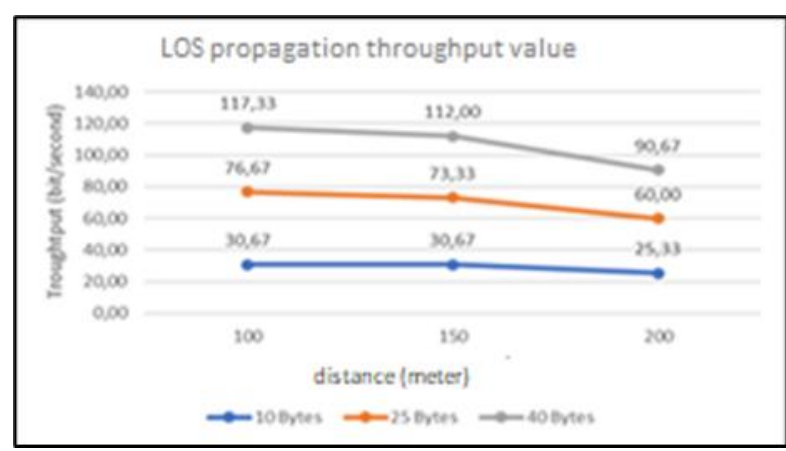

Figure 12. Throughput Graph on LOS Propagation Measurement

\section{Testing Throughput on NLOS Propagation}

Throughput on NLOS propagation is measured at 100, 150, and 200 meters by sending as much data as possible from the end device to the Application Server in 1 minute with packet sizes of 10, 25, and 40 Bytes. The measurement results at each distance are then converted into bits and divided by the time used for data transmission, which is 1 minute or 60 seconds, to yield a result in bits per second. Figure 13 depicts the results of throughput testing on NLOS propagation.

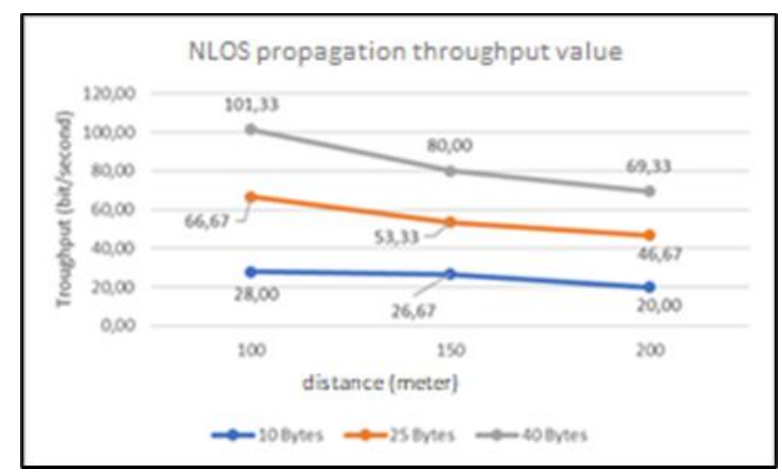

Figure 13. Throughput Graph on NLOS Propagation Measurement

According to the graph in Figure 13, the larger the packet sent, the higher the Throughput obtained. The lower the throughput, the greater the measurement distance. According to the measurement results, the throughput value in NLOS propagation is less than the throughput value in LOS propagation at each distance and packet size.

\section{CONCLUSION}

Based on the findings of the study, it is possible to conclude that the success rate of LOS propagation measurements is higher than that of NLOS propagation. At a distance of 100 meters, the LOS value is $17 \%$ greater than the NLOS value. At a distance of 150 meters, the LOS value is $24 \%$ greater than the NLOS value. The LOS value is $3 \%$ greater than the NLOS value at a distance of 200 meters. For each amount of data sent and measurement distance, the throughput value in LOS propagation measurement is greater than that in NLOS propagation.

\section{ACKNOWLEDGEMENT}

The author wishes to express his gratitude to IT Telkom Purwokerto for its moral and material support in conducting this research and publication.

\section{REFERENCES}

[1] Shintaloka Pradita Sicca, "BPS: Jumlah Penduduk Bekerja Triwulan I 2018 Sebanyak 127,07 Juta," tirto.id, 2018. [Online]. Available: https://tirto.id. [Accessed: 17-Jun2019].

[2] H. Husdi, "Monitoring Kelembaban Tanah Pertanian Menggunakan Soil Moisture Sensor Fc-28 Dan Arduino Uno,” Ilk. J. Ilm., vol. 10, no. 2, p. 237, 2018.

[3] N. Ducrot, D. Ray, A. Sadani, O. Hersent, G. Pop, and G. Remond, "LoRa Device Developer Guide," 2016.

[4] LoRa Alliance, "LoRaWAN - What is it?. A technical overview of LoRa and LoRaWAN," 2015.

[5] W. P. Putra, R. I. M, A. Sumarudin, and A. E. Putro, "Implementasi Lorawan Server Untuk Sistem Tracking Perahu Nelayan Berbasis MQTT Protocol," J. Appl. Informatics Comput., vol. 2, no. 2, pp. 46-50, 2018.

[6] Polymorph, "IoT connectivity comparison (GSM vs LoRa vs Sigfox vs NB-Iot)," 2018. [Online]. Available: https://www.polymorph.co.za/iot-connectivitycomparison-gsm-vs-lora-vs-sigfox-vs-nb-iot/\#).

[7] F. N. Aroeboesman, M. H. H. Ichsan, and R. Primananda, "Analisis Kinerja LoRa SX1278 Menggunakan Topologi Star Berdasarkan,” JPTIK, vol. 3, no. 4, pp. 3860-3865, 2019.

[8] A. J. Wixted, P. Kinnaird, H. Larijani, A. Tait, A. Ahmadinia, and N. Straxhman, "Evaluation of LoRa and LoRaWAN for Wireless Sensor Network," IEEE SENSORS, vol. 3, no. May, pp. 31-48, 2016.

[9] M. Diana, R. Nazir, and A. Rufiyanto, "Harvesting RF Ambient Energy dari End Device LoRa (Long Range Access)," J. Infotel, vol. 9, no. 4, p. 387, 2018.

[10] R. Hartono and A. Purnomo, "Wireless Network 802.11," Surabaya, 2011.

[11] I. Dinata and W. Sunanda, "Implementasi Wireless Monitoring," J. Nas. Tek. Elektro, vol. 4, no. 1, pp. 83-88, 2015.

\section{AUTHOR BIOGRAPHY}

Fikri Nizar Gustiyana, is a Telecommunication Engineering student at Institut Teknologi Telkom Purwokerto. The author completed his studies at the Institut Teknologi Telkom Purwokerto in 2020. The author's interest in the field of Internet Of Things. 
Muntaqo Alfin Amanaf, is a lecturer in the Department of Telecommunications Engineering, Telkom Institute of Technology Purwokerto. Diploma 4 studies were completed at PENS-ITS Surabaya and postgraduate studies were completed at the Institut Teknologi Sepuluh Surabaya. The author's research field is in the field of wireless cellular, wireless technology and wireless sensor network.

Danny Kurnianto, is a lecturer in the Department of Telecommunication Engineering, Institut Teknologi Telkom Purwokerto. Undergraduate studies were completed at Brawijaya University Malang and master studies were completed at Gadjah Mada University Yogyakarta. The author's research fields are in the fields of Internet of Things, Embedded Systems and Signal Processing. 